\title{
Characteristic features and biotechnological applications of cross-linked enzyme aggregates (CLEAs)
}

\author{
Roger A. Sheldon
}

Received: 11 July 2011 /Revised: 8 August 2011 / Accepted: 13 August 2011 /Published online: 2 September 2011

(C) The Author(s) 2011. This article is published with open access at Springerlink.com

\begin{abstract}
Cross-linked enzyme aggregates (CLEAs) have many economic and environmental benefits in the context of industrial biocatalysis. They are easily prepared from crude enzyme extracts, and the costs of (often expensive) carriers are circumvented. They generally exhibit improved storage and operational stability towards denaturation by heat, organic solvents, and autoproteolysis and are stable towards leaching in aqueous media. Furthermore, they have high catalyst productivities (kilograms product per kilogram biocatalyst) and are easy to recover and recycle. Yet another advantage derives from the possibility to co-immobilize two or more enzymes to provide CLEAs that are capable of catalyzing multiple biotransformations, independently or in sequence as catalytic cascade processes.
\end{abstract}

Keywords Immobilization · Hydrolases · Oxidoreductases . Lyases $\cdot$ Magnetic $\cdot$ Sustainable

\section{Introduction}

One of the great challenges that the pharmaceutical, chemical, and allied industries face in the 21 st century is the transition to a greener and more sustainable manufacturing base that minimizes, or preferably avoids, the generation of waste and the use of toxic and/or hazardous materials. Biocatalysis has many benefits to offer in this respect. Reactions can be performed in conventional

\section{R. A. Sheldon $(\triangle)$}

Department of Biotechnology, Delft University of Technology, Julianalaan 136 ,

NL-2628 BL Delft, The Netherlands

e-mail: r.a.sheldon@tudelft.nl reactors (no specialized equipment is needed) under mild conditions (ambient temperature and pressure, physiological $\mathrm{pH}$ ) in an environmentally acceptable solvent (water) using a biocompatible and biodegradable catalyst (an enzyme) that is itself derived from renewable resources.

Enzymatic reactions proceed with high regio- and stereoselectivity and generally without the need for functional group activation and protection and deprotection steps required in traditional syntheses. Hence, generally speaking, enzymatic processes generate less waste than conventional synthetic routes, are more energy efficient, and provide products in higher purity. The application of modern protein engineering techniques, such as directed evolution, has enabled the optimization of their properties to fit pre-defined process parameters (Luetz et al. 2008; Turner 2009). In short, enzymes can be "tailor made" and can be produced on a large scale for an attractive price.

Notwithstanding all these advantages, enzymes have some drawbacks that have limited their widespread application. They often lack operational and storage stability, for example. Enzymes are complex, highly sensitive molecules with unique three-dimensional structures that are essential for their activities. Exposure to certain conditions, such as elevated temperatures or organic solvents, can lead to denaturation (unfolding) and concomitant loss of activity. Furthermore, enzymes are generally used as aqueous solutions which makes recovery and reuse problematical and can also result in contamination of the product. These obstacles can generally be overcome by immobilization of the enzyme, affording improved storage and operational stability and providing for its facile separation and reuse (Sheldon 2007a). Moreover, immobilized enzymes, in contrast to free enzymes which can penetrate the skin, are unlikely to cause allergic reactions. 


\section{Enzyme immobilization: an enabling technology}

Immobilization can enable economically viable applications of enzymes that, for one or more of the reasons outlined above, would not have been viable using the free enzyme. It typically involves binding the enzyme to a carrier (support), such as an ion exchange resin or silica, or encapsulation in an inert carrier, affording a biocatalyst with superior operational performance compared to the free enzyme. Binding to the carrier can involve simple adsorption, e.g., via hydrophobic or ionic interactions or actual covalent bonding. A serious drawback of such carrierbound enzymes (Cao et al. 2003) in general is their low productivities (kilograms product per kilogram enzyme) owing to the large amount of non-catalytic ballast (often $>95 \%$ of the total mass). They can also be costly as some of the carriers that are used are themselves expensive materials. In contrast, immobilization by cross-linking of enzyme molecules affords carrier-free immobilized enzymes with high productivities and avoids the costs of the carrier.

Cross-linked enzymes, produced by mixing an aqueous solution of the enzyme with an aqueous solution of glutaraldehyde, were already known in the 1960s but were generally difficult to handle, gelatinous materials exhibiting low activity, poor reproducibility, and low stability and shelf life. Consequently, carrier-bound enzymes became the method of choice for the next three decades. In the early 1990s, Altus Biologics introduced the use of cross-linked enzyme crystals (CLECs) as industrial biocatalysts (Margolin and Navia 2001). The methodology was applicable to a wide variety of enzymes, and CLECs exhibited excellent operational stability, controllable particle size coupled with high productivity, and facile recovery and reuse, making them ideally suited to industrial biocatalysis. However, they had one inherent limitation: the need to crystallize the enzyme, a laborious procedure requiring enzyme of high purity which meant relatively high costs.
Several years ago, we reasoned that crystallization could perhaps be replaced by precipitation of the enzyme from aqueous buffer, a simpler and less expensive method not requiring highly pure enzymes. It is well documented that the addition of salts, or water-miscible organic solvents or non-ionic polymers, to aqueous solutions of proteins leads to their precipitation as physical aggregates that are held together by non-covalent bonding (Burgess 2009). Addition of water to this precipitate results in dissolution of the enzyme. In contrast, we reasoned that cross-linking of these physical aggregates, to form what is essentially a crosslinked polymer, would render them permanently insoluble while maintaining their pre-organized superstructure and hence their catalytic activity. This led us to develop (Cao et al. 2000; Sheldon 2011) a new class of immobilized enzymes which we called cross-linked enzyme aggregates (CLEAs). Since selective precipitation with ammonium sulfate is a commonly used method for enzyme purification (Englard and Seifter 1990), the CLEA methodology essentially combines two unit processes, purification and immobilization, into a single operation. In principle, one can even take the crude enzyme extract from fermentation broth and produce the immobilized enzyme in one simple operation. Hence, one can envisage the direct immobilization of an enzyme, at the production site, without any need for intermediate work-up or purification.

\section{Preparation of CLEAs}

A general scheme for the preparation of a CLEA is outlined in Fig. 1. Glutaraldehyde is generally the cross-linking agent of choice as it is inexpensive and readily available in commercial quantities. It has been used for decades for cross-linking proteins. However, the chemistry is complex and not fully understood. Cross-linking occurs via reaction of the free amino groups of lysine residues, on the surface of neighboring enzyme molecules, with oligomers or

Fig. 1 General scheme for CLEA preparation

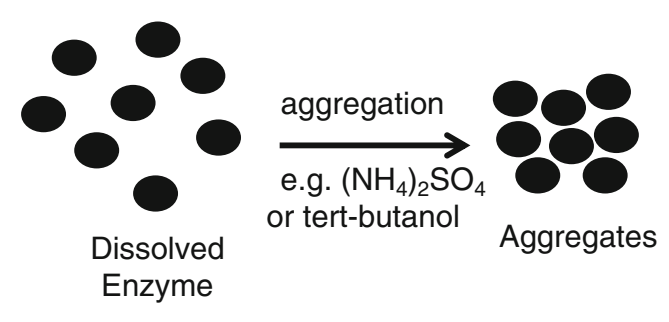

Enzyme

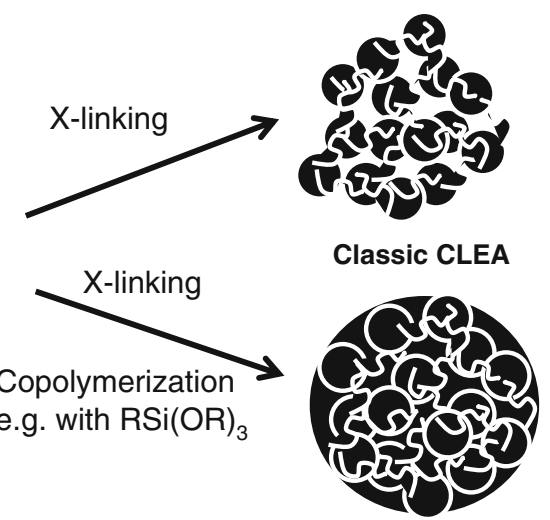

Silica-CLEA composite 
polymers of glutaraldehyde resulting from inter- and intramolecular aldol condensations. Cross-linking can involve both Schiff's base formation and Michael-type 1,4 addition to $\alpha, \beta$-unsaturated aldehyde moieties, and the exact mode of cross-linking is $\mathrm{pH}$ dependent (Migneault et al. 2004; Walt and Agayn 1994). Other dialdehydes, involving less complicated chemistry, can be used as cross-linkers, e.g., dextran polyaldehyde, followed by reduction of the Schiff's base moieties with sodium cyanoborohydride to form irreversible amine linkages (Mateo et al. 2004).

A variation on this theme involves performing the crosslinking in the presence of a monomer that undergoes polymerization under these conditions. This results in the formation of CLEA-polymer composites with tunable physical properties. For example, if the cross-linking is performed in the presence of a siloxane, e.g., $(\mathrm{MeO})_{4} \mathrm{Si}$, the latter undergoes simultaneous polymerization to afford a CLEA-silica composite (Schoevaart et al. 2006). The hydrophobic/hydrophilic properties and particle size of the latter can be tailored by manipulating the structure of the siloxane used. More recently, we have made "smart" magnetic CLEAs by conducting the cross-linking in the presence of functionalized magnetic nanoparticles. These mCLEAs can be separated by magnetic decantation or can be used in a magnetically stabilized fluidized bed, and we envisage that this will lead to novel combinations of bioconversions and downstream processing.

Optimization of the protocols for CLEA preparation, with regard to parameters such as temperature, $\mathrm{pH}$, concentration, stirring rate, precipitant, additives, and cross-linking agent, is a relatively simple operation which lends itself to automation, e.g., using 96-well plates. The nature of the precipitant predictably has an important effect on the activity recovery, and hence, it is necessary to screen a number of water-miscible organic solvents and polymers such as polyethylene glycols. In the initial screening of precipitants, the amount of aggregates formed is determined, and then they are redissolved in aqueous buffer and their activities measured. However, in our experience, observation of a high activity on redissolution of the precipitate in buffer does not automatically mean that the aggregates will retain this high activity after cross-linking. Hence, the optimum precipitant may not be the one that ultimately gives the optimum CLEA. It is advisable, therefore, to choose a few precipitants which give good yields of aggregates for further screening in subsequent cross-linking.

The molar ratio of cross-linker to enzyme is important as it influences the activity, stability, and particle size of the resulting CLEA. If the ratio is too low, sufficient crosslinking does not occur, affording a CLEA that is too flexible and unstable towards leaching in water. If it is too high, too much cross-linking can result in a complete loss of the enzyme's flexibility and hence its activity. Since every enzyme has a unique surface structure, containing varying numbers of lysine residues, the optimum ratio has to be determined for each enzyme. With enzymes containing few or no accessible lysine residues, cross-linking may be insufficient and lead to CLEAs that are unstable towards leaching in aqueous media. One way to overcome this problem is to add polyamines, such as polyethyleneimine, which are then co-immobilized with the enzyme (LopezGallego et al. 2005). Problems can also be encountered in CLEA formation when the protein concentration in the enzyme preparation is low. In such cases, CLEA formation can be promoted by the addition of a second protein, such as bovine serum albumin, as a so-called proteic feeder (Shah et al. 2006).

A key, cost-determining parameter of a CLEA is the activity recovery, which should preferably be close to (or more than) $100 \%$. Ultimately, the important commercial parameter, enzyme costs per kilogram of product, is determined by how often the CLEA can be recycled. Activity recovery is simply total activity in the CLEA divided by the activity of the free enzyme charged, expressed as a percentage, and is independent of the weight of the CLEA. Thus, $100 \%$ activity recovery could be obtained with an increase or a decrease in weight of the CLEA compared to that of the free enzyme sample charged. Specific activity, on the other hand, is defined as an activity per unit weight of protein. Obviously, the activity of the free enzyme charged and that of the immobilized enzyme recovered need to be measured under the same conditions. This is relatively straightforward for a hydrolytic process in which the free enzyme is dissolved in water, e.g., tributyrin hydrolysis for a lipase. However, comparison becomes more problematical for a synthesis reaction in non-aqueous media where the free enzyme is not soluble. In this case, many authors compare the specific activity of the immobilized lipase with that of so-called acetone powder, obtained by precipitation of the enzyme from aqueous solution with acetone. Another possibility is to compare it with freeze-dried enzyme.

\section{Characteristic features of CLEAs}

\section{Physical properties}

Particle size is an important property of any heterogeneous catalyst in the context of industrial applications since it directly effects mass transfer and filterability under operational conditions. A typical particle size of CLEAs is $5-50 \mu \mathrm{m}$, and their filterability is generally sufficient for batch operation. As with all solid catalysts, 
one might expect loss of activity owing to diffusional limitations in an immobilized enzyme. However, CLEAs are highly porous materials, and we have generally not observed any diffusional limitations when using them in biocatalytic transformations commonly used in organic synthesis. In contrast, we have observed diffusional limitations in colorimetric assays which are usually very fast reactions. Consequently, activities based on such assays should be treated with caution as they may lead to the erroneous conclusion that the CLEA has a low activity in the envisaged biotransformation.

Obviously, the rate of diffusion is influenced by the particle size, and this is determined by, inter alia, the crosslinker/enzyme ratio. Optimum rates are observed with smaller particles, but practical considerations, e.g., ease of filtration, dictate the use of larger particles. This necessitates a compromise between good activity and good filterability. Particle size is influenced by certain parameters in the synthesis, such as cross-linker-to-enzyme ratio (Yu et al. 2006). The particle size of a CLEA can also increase on aging (Pchelintsev et al. 2009) which strongly suggests that the cross-linking process was not complete when the reaction was stopped. However, continuous operation over a packed bed of biocatalyst will require relatively large particles in order to avoid a large pressure drop over the column. One approach to preparing CLEAs with increased particle size and enhanced mechanical stability is to encapsulate them in a polyvinyl alcohol matrix (so-called Lentikats). This results in a minor dilution of activity that was deemed acceptable (Wilson et al. 2004a).

One can envisage the use of smaller particles in a fluidized bed which requires particles of sufficient density that they are not blown out of the column. Alternatively, magnetic CLEAs can be held in place in a magnetically stabilized bed. Similarly, other physical attributes that effect recovery, handling, and recyclability, such as hardness, density, porosity, and robustness of the CLEA particles, can be modified by preparing the silica-CLEA composites (see earlier) with varying hydrophobicity and particle size.

\section{Operational stability}

The main reason for immobilizing an enzyme is probably the significant enhancement of operational stability towards heat and organic solvents that is generally achieved. This is a direct result of the decrease in flexibility which suppresses the propensity of enzymes towards unfolding and accompanying loss of tertiary structure necessary for activity. Indeed, we have found that immobilization of enzymes as cross-linked enzyme aggregates can lead to dramatic increases in operational stability at elevated temperatures. For instance, the limited thermal stability of papain (the protease from Carica papaya) is well docu- mented. As shown in Fig. 2, the stability of a papainCLEA, in $N$-Bz-Arg-OEt hydrolysis at $\mathrm{pH} 7$ and $50^{\circ} \mathrm{C}$, was dramatically enhanced compared to the free papain (Sorgedrager et al. (unpublished results)).

A characteristic feature of many multimeric enzymes is their lack of thermal stability outside the cell owing to facile multimer dissociation with concomitant loss of activity. Nitrile hydratases (NHases), for example, constitute a class of industrially important enzymes (DiCosimo 2006; van Pelt et al. 2008a) that are notoriously unstable outside the cell. Consequently, NHase-mediated industrial biotransformations, such as acrylamide and nicotinamide manufacture from the corresponding nitriles, are limited to whole cell processes. We found that immobilization of a nitrile hydratase from an alkaliphilic bacterium, Nitriliruptor akaliphilus, as a CLEA led to a dramatic increase in storage stability compared to the free enzyme as shown in Fig. 3 (van Pelt et al. 2008b).

\section{Recyclability}

Another important reason to immobilize an enzyme is to facilitate its recovery from the reaction medium and subsequent reuse, resulting in simplification of downstream processing and reduction in enzyme cost contribution to the product. In our experience, CLEAs are eminently recyclable. For example, the effect of recycling on the performance of the (R)-oxynitrilase-CLEA from Prunus amygdalus in the hydrocyanation of $o$-chlorobenzaldehyde is illustrated in Fig. 4. The CLEA was recycled ten times with negligible loss in activity (van Langen et al. 2005).

Similarly, the NHase-CLEA described above was recycled 36 times in the hydration of n-hexyl cyanide. The observed loss of selectivity can probably be largely attributed to mechanical losses that inevitably occur on pipetting the supernatant from the solid CLEA (van Pelt et al. 2008b).

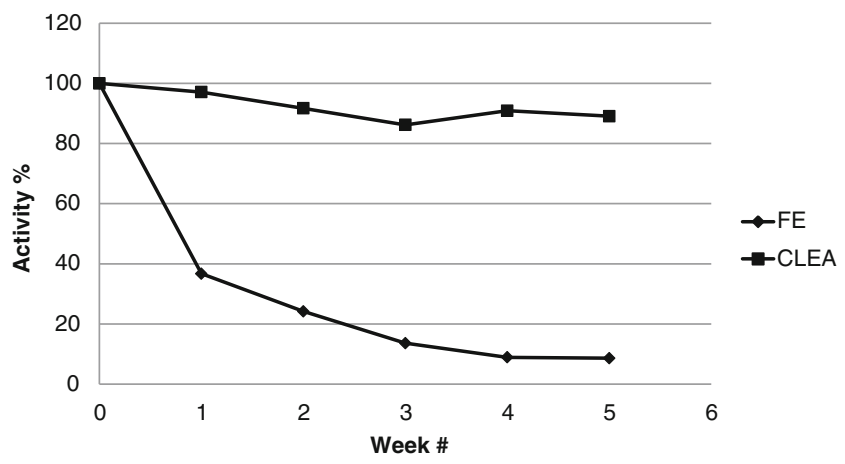

Papain (protease from C. papaya) incubated at $\mathrm{pH} 7$ and $50^{\circ} \mathrm{C}$. Residual activity in N-Bz-Arg-OEt hydrolysis

Fig. 2 Thermal stability of papain-CLEA 


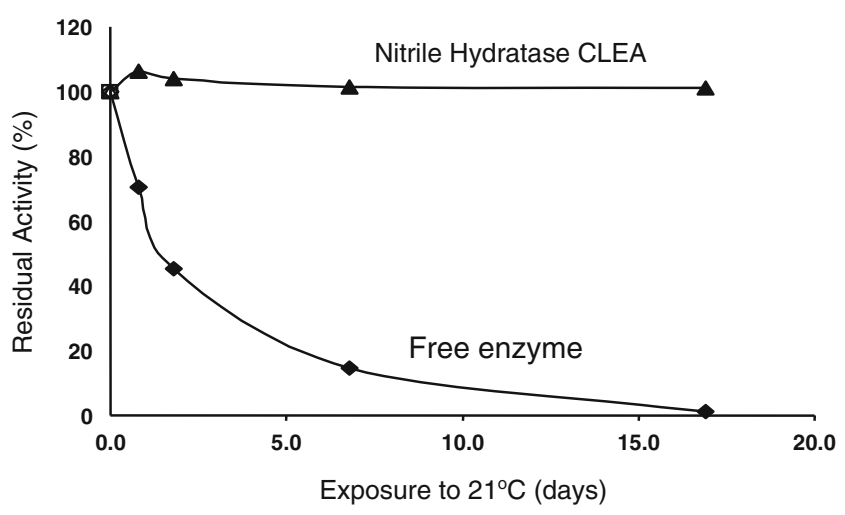

Fig. 3 Storage stability of NHase at $21^{\circ} \mathrm{C}$

\section{Reactor design}

The application of immobilized enzymes in the pharmaceutical and fine chemical industries generally involves batch processes with recovery by filtration or centrifugation. In other industries, such as the conversion of oils and fats, a packed bed of CLEA in continuous operation mode may be the method of choice. This generally requires a compromise with regard to particle size to avoid the large pressure drop over the column observed with small particles without the lower activities due to diffusion limitations in large particles. Alternatively, a fluidized bed of small particles can be used, but they must be relatively dense. We have also described the successful use of a penicillin amidase CLEA in a so-called filter slurry reactor for the continuous hydrolysis of penicillin $\mathrm{G}$ (Sorgedrager et al. 2008). An interesting recent elaboration of the CLEA methodology is the development of magnetic CLEAs by performing the cross-linking in the presence of functionalized magnetic nanoparticles (unpublished results). The resulting magnetic CLEAs can be separated by magnetic
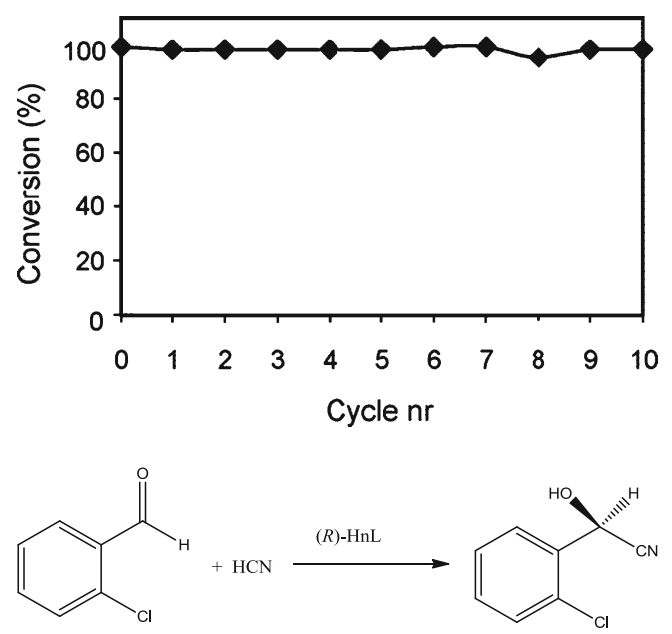

Fig. 4 Recyclability of (R)-hydroxynitrile lyase-CLEA in $o$-chlorobenzaldehyde hydrocyanation decantation or can be used in a magnetically stabilized fluidized bed. The use of CLEAs in microchannel reactors has also been described (Hickey et al. 2007).

\section{Scope of the technology}

The CLEA technology has broad scope (Sheldon 2007b) and has been applied to an increasingly wide selection of hydrolases, oxidoreductases, and lyases (see Table 1). The majority of CLEAs that have been described involve hydrolases, probably because they are the enzymes that have the most industrial applications and are the simplest enzymes to work with. Multipurpose CLEAs can be prepared from crude enzyme extracts consisting of multiple enzymes. For example, a multi-CLEA prepared from a porcine pancreatic acetone powder, containing lipase, phospholipase $\mathrm{A}_{2}$, and $\alpha$-amylase enzymes, retained all three enzyme activities (Dalal et al. 2007). In contrast, we use the term combi-CLEA for deliberate co-immobilization of two or more enzymes in a single CLEA for the sole purpose of performing two or more biotransformations in a multi-enzyme cascade process (see later for examples).

\section{Hydrolase CLEAs}

\section{Protease CLEAs}

We have prepared CLEAs from a variety of proteases, including (chymo)trypsin (E.C. 3.4.21.1), papain (E. C.3.4.22.2), and the inexpensive alkaline protease from Bacillus licheniformis (alcalase, E.C. 3.4.21.62, also known as subtilisin Carlsberg) used in laundry detergents. Alcalase has been widely used in organic synthesis, e.g., in the resolution of (amino acid) esters, and amines and peptide

Table 1 Examples of enzymes that have been successfully cleated

\begin{tabular}{lll}
\hline Hydrolases $^{\mathrm{a}}$ & Oxidoreductases $^{\mathrm{a}}$ & Lyases $^{\mathrm{a}}$ \\
\hline Lipases (18) & Keto reductase & Hydroxynitrile lyases (6) \\
Esterases (3) & Formate dehydrogenase & Pyruvate decarboxylase \\
Proteases (7) & Glucose oxidase & Deoxyribose aldolase \\
Nitrilases (2) & Galactose oxidase & Nitrile hydratase \\
Amidases (4) & Amino acid oxidase & \\
Phytase & Laccase & Transferases \\
Galactosidase & Catalase & Transaminases \\
Carbonic & Chloroperoxidase & \\
anhydrase & Horse radish peroxidase & \\
Amylase & H. & \\
\hline
\end{tabular}

${ }^{a}$ Figures in parentheses represent the number of enzymes in this class that have been successfully cleated 
synthesis. We have demonstrated that the alcalase-CLEA has excellent storage and operational stability. It is not surprising, therefore, that alcalase-CLEA has been used in the synthesis of amino acid derivatives and peptides in organic solvents, notably methyl tert-butyl ether (Nuijens et al. 2010).

A novel application of protease-CLEAs, for which longterm stability is essential, is as an environmentally acceptable substitute for organotin anti-fouling agents in paint used in marine applications. The use of toxic organotin reagents has been banned in the EU since 2008. The stabilities of CLEAs of proteases were tested in artificial seawater (ASW) both as it is and as a component of the paint (Skovgaard et al. 2010). All of the protease CLEAs showed excellent tolerance to xylene and had great stability in dried paint. Furthermore, the alcalase-CLEA exhibited $900 \%$ activation during storage in ASW in dried paint. In contrast, the free alcalase lost more than $90 \%$ of its activity in 28 days in the paint. This would appear to offer great potential for the application of proteaseCLEAs and perhaps CLEAs of other enzymes as anti-fouling agents in a variety of coatings.

\section{Amidase CLEAs}

Our initial studies of CLEAs were concerned with penicillin $G$ amidase (E.C. 3.5.1.11), an industrially important enzyme used in the synthesis of semi-synthetic penicillin and cephalosporin antibiotics. The limited thermal stability and low tolerance to organic solvents of the free enzyme make it an ideal candidate for stabilization as a CLEA. Indeed, penicillin $G$ amidase CLEAs have been shown to be extremely effective catalysts, with high volumetric productivities for the hydrolysis of penicillin $G$ and the synthesis of semi-synthetic penicillins (Cao et al. 2000, 2001; Illanes et al. 2006) and cephalosporins (Illanes et al. 2007, 2009; Wilson et al. 2009) such as ampicillin and cephalexin, respectively (see Fig. 5).

The aminoacylase (EC 3.5.1.14) derived from Aspergillus melleus is used in the Evonik process for the manufacture of $(S)$-amino acids such as $(S)$-methionine by highly enantioselective hydrolysis of the corresponding $N$-acetyl amino acid (Fig. 6). We prepared a CLEA from this enzyme and showed it to be an active and recyclable catalyst for this reaction (Bode et al. 2003). Similarly, Dong and coworkers prepared an $A$. melleus aminoacylase CLEA by coprecipitation with bovine serum albumin (BSA) as a proteic feeder and crosslinking with glutaraldehyde (Dong et al. 2010). They observed $82 \%$ activity recovery, compared to $24 \%$ observed in the absence of added BSA, which was attributed to the low number of surface amino groups in the enzyme. The aminoacylase-BSA-CLEA retained more than $80 \%$ of its activity after ten cycles in the enantioselective hydrolysis of racemic $N$-acetyl methionine, demonstrating its robustness under operational conditions.

A CLEA was prepared from a crude cell-free extract of Rhodococcus erythropolis amidase using BSA as a proteic feeder and cross-linking with glutaraldehyde (Park et al. 2010). It catalyzed the enantioselective hydrolysis of 4chloro-3-hydroxybutyramide, albeit in low enantioselectivity (60\% enantiomeric excess (ee) at $65 \%$ conversion).

\section{Lipase and esterase CLEAs}

Lipases (E.C. 3.1.1.3) are used in a broad palette of industrial applications. It is not surprising, therefore, that a variety of lipase-CLEAs have been prepared and used as

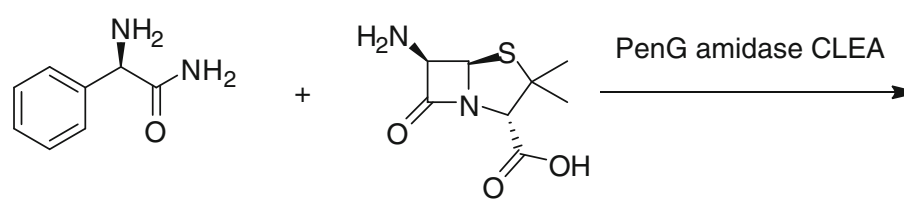

6-APA<smiles>CC1(C)S[C@@H]2[C@H](NC(=O)[C@@H](N)c3ccccc3)C(=O)N2[C@H]1C(=O)O</smiles>

Ampicillin<smiles>COC(=O)[C@H](N)c1ccccc1</smiles>

7-ADCA<smiles>CO[N+]1=C(C(=O)O)C2C(=O)C(NC(=O)[C@H](N)c3ccccc3)[C@H]2SC1</smiles>

Cephalexin

Fig. 5 Synthesis of ampicillin and cephalexin with pen G amidase-CLEA 


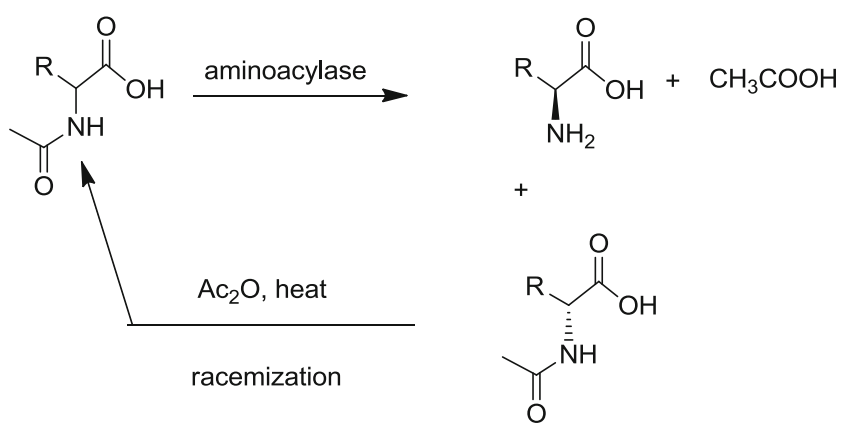

Fig. 6 Aminoacylase catalyzed resolution of $N$-acylamino acids

robust, recyclable catalysts in organic synthesis (LopezSerrano et al. 2002). For example, Candida antarctica lipase B (CaLB) is widely used in industrial organic synthesis, and we have shown that CaLB-CLEA is completely stable towards leaching in aqueous media. Furthermore, CaLB-CLEAs exhibited performed admirably in organic solvents and in supercritical carbon dioxide (Hobbs et al. 2006).

Similarly, lipase-CLEAs have been prepared from, inter alia, C. antarctica lipase A (Özdemirhan et al. 2008), Burkholderia cepacia (formerly known as Pseudomonas cepacia) (Kumari et al. 2007; Majumder et al. 2008; Hara et al. 2008), Candida rugosa (Yu et al. 2006; Kartal et al. 2011), and a Pseudomonas sp. lipase (Zhao et al. 2008). ${ }^{1}$

In contrast to the extensive studies devoted to lipase CLEAs, little attention has been paid to other esterases. CLEAs have been prepared from feruloyl esterase (E.C. 3.1.1.73) (Vafiadi et al. 2008) and acetylxylan esterase (Montoro-Garcia et al. 2009), enzymes that play a key role in the degradation of lignocellulose and, consequently, are of commercial importance in the context of biomass conversion processes. In vitro, the latter enzyme catalyzes the hydrolysis of the acetate moiety in cephalosporin $\mathrm{C}$ and 7-aminocephalosporanic acid to form advanced intermediates for the production of semi-synthetic cephalosporins (Montoro-Garcia et al. 2009).

\section{Glycosidase CLEAs}

Glycosidases constitute a diverse and industrially very important class of enzymes that play a vital role in carbohydrate chemistry, for example in the in vivo hydrolysis of biopolymers such as starch, inulin, and (lingo) cellulose. They include, for example, amylases, xylanases, pectinases, and cellulases. The latter are currently the focus of much attention in connection with the pretreatment of lignocellulose in the manufacture of second generation biofuels from biomass. CLEAs have been

\footnotetext{
$\overline{{ }^{1} \text { Lipase-CLEA }}$ discovery platforms can be obtained from CLEA Technologies (www.cleatechnologies.com).
}

successfully prepared from selected glycosidases. For example, we have successfully prepared a galactosidaseCLEA (Schoevaart et al. 2004) with excellent operational stability and recyclability for use in the hydrolysis of lactose in dairy products and the conversion of lactose to lactulose or the synthesis of galacto-oligosaccharides for use as prebiotic food additives.

\section{Oxidoreductase CLEAs}

\section{Oxidase CLEAs}

Glucose oxidase (GOD; E.C. 1.1.3.4) is a flavin-dependent oxidase that catalyzes the aerobic oxidation of D-glucose to gluconic acid with concomitant production of one equivalent of hydrogen peroxide (Bankar et al. 2009). GOD is exploited in a variety of industrial applications including glucose removal from dried egg; oxygen removal from fruit juices, canned beverages, and mayonnaise; and improvement of color, flavor, and shelf life of foods. It is also used in conjunction with catalase in many of these applications and in biosensors for the detection of glucose in body fluids and industrial solutions. Most commercially available GOD is produced from Aspergillus niger and is essentially a byproduct of gluconic acid production. As GOD is often used in conjunction with catalase, co-immobilization of GOD with catalase has been the subject of extensive investigation. Consequently, we prepared a combi-CLEA, by coprecipitation of the two enzymes and cross-linking with glutaraldehyde, and showed that it had excellent activity retention and could be recycled without loss of activity (Schoevaart et al. 2004). Similarly, we prepared a combi-CLEA from galactose oxidase (a copper-dependent oxidase) and catalase and showed that it was effective in catalyzing the aerobic oxidation of D-galactose to the corresponding 6-aldehyde (Schoevaart et al. 2004).

Another interesting oxidase, from the viewpoint of industrial applications, is the multicopper oxidase, laccase (polyphenoloxidase, EC 1.10.3.2), a lignin-modifying enzyme that catalyzes the oxidation of phenolic compounds and aromatic amines. Over the last two decades the use of laccase has been explored for the bioremediation of xenobiotics such as endocrine-disrupting compounds (EDCs), for bleaching in the pulp and paper industry, and for decolorization of azo dyes in the textile industry. However, the application of laccase in free form (in solution) for the treatment of EDCs and azo dyes is not economically viable owing to poor operational stability and lack of reusability. One approach to overcoming these obstacles and hence to enabling an economically viable technology is to immobilize the laccase. Immobilization of laccase on solid supports significantly enhances its stability towards denaturation 
under operating conditions and allows for continuous processing but, at the same time, results in low productivities owing to the large percentage of non-catalytic mass in the catalyst. In stark contrast, immobilization of laccase as CLEAs affords an immobilized catalyst that combines enhanced operational stability and reusability with productivities close to those of the free enzyme (Schoevaart et al. 2006; Matijosyte et al. 2010). This provides a sound basis for the development of cost-effective, laccase-based processes for use in industrial and environmental biotechnology applications (Cabana et al. 2007).

Furthermore, laccase can be used in conjunction with a mediator, commonly the stable free radical, TEMPO, or a derivative thereof, for the oxidation of simple alcohols. For example, laccase/TEMPO catalyzes the selective aerobic oxidation of starch to carboxystarch (Fig. 7). The latter is of interest as an environmentally friendly water superabsorbent to replace the poorly biodegradable polyacrylates currently used. Unfortunately, the enzyme costs are prohibitively high as a result of the poor stability of the laccase which undergoes suicide inactivation under the oxidizing conditions. We reasoned that the stability of laccase under operating conditions could probably be improved by removing reactive amino groups on the enzyme surface by cross-linking with glutaraldehyde. Indeed, we prepared laccase-CLEAs and showed that they had enhanced operational stability compared to the free enzymes (Schoevaart et al. 2006), thus paving the way for an economically viable aerobic oxidation of starch to carboxystarch.

Tyrosinase (E.C. 1.14.18.1) is a copper-dependent oxidase with properties very similar to laccase. It catalyzes the ortho-hydroxylation of phenols and has potential applications in the same areas as for laccase. A CLEA was prepared from mushroom tyrosinase with $100 \%$ activity recovery, via precipitation with ammonium sulfate and cross-linking with glutaraldehyde (Aytar and Bakir 2008). It exhibited enhanced thermal stability, both on storage and under operational conditions, compared to the free enzyme.

\section{Peroxidase CLEAs}

An additional benefit of the CLEA technology is that it can stabilize the quaternary structures of multimeric enzymes, a common structural feature of many heme peroxidases, towards dissociation and loss of activity. For example, the stability of CLEAs from two tetrameric catalases (E.C. 1.11.1.6) exhibited improved stability compared to the free enzyme (Wilson et al. 2004b). Similarly, the thermal stability of a CLEA prepared from royal palm (Roystonea regia) peroxidase was 5,000-fold better than the free enzyme. The peroxidase CLEA could be used several times in the decolorization of wastewater containing azo dyes. A CLEA was also successfully prepared from horse radish peroxidase with $83 \%$ activity recovery (Sulek et al. 2011). Potential applications are foreseen in the biosensor field.

The heme-dependent chloroperoxidase (CPO, E. C.1.11.1.10) from Caldariomyces fumago catalyzes in vivo the oxidation of chloride ion to hypochlorite, by hydrogen peroxide. In the absence of chloride ion, it catalyzes a variety of regio- and enantioselective oxygen transfer processes of synthetic interest. However, a major drawback of CPO is its low stability owing to the facile oxidative degradation of the porphyrin ring by hydrogen peroxide even at relatively low peroxide concentrations. A CLEA was successfully prepared from CPO in $68 \%$ activity recovery under optimized conditions (Perez et al. 2009). It exhibited enhanced thermal stability and tolerance towards hydrogen peroxide during the sulfoxidation of anisole.

\section{Lyase CLEAs}

\section{Nitrile hydratase CLEAs}

NHases (E.C. 4.2.1.84) are Fe- or Co-dependent metalloenzymes that catalyze several industrially important pro-

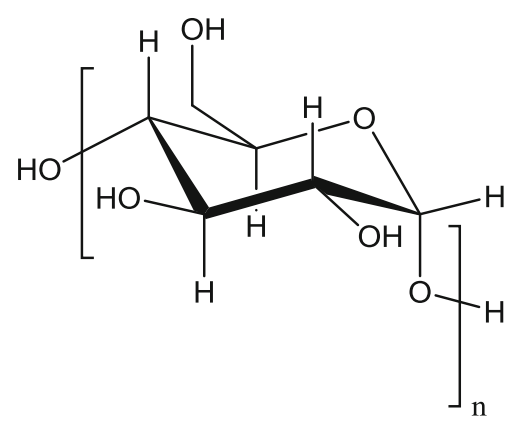

starch

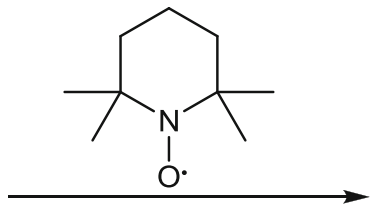

laccase $/ \mathrm{O}_{2}$

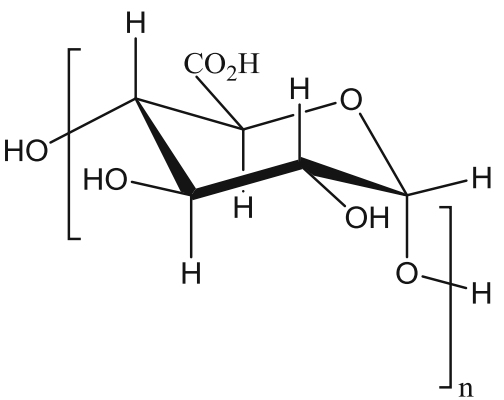

carboxystarch

Fig. 7 Aerobic oxidation of starch to carboxystarch catalyzed by laccase/TEMPO 


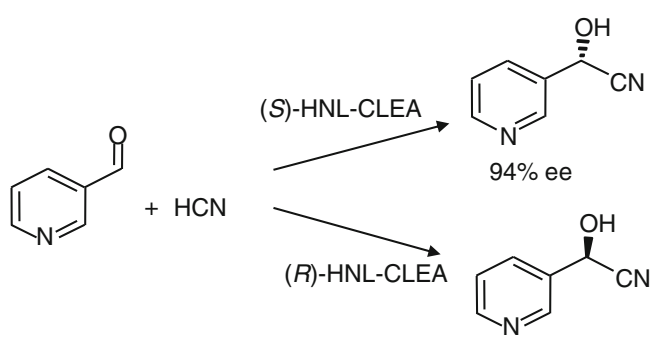

( $93 \%$ ee)

Fig. 8 Enantioselective hydrocyanation of pyridine 3-aldehyde with HNL-CLEAs

cesses involving the hydration of nitriles to the corresponding amides (van Pelt et al. 2008a). They are often multimeric enzymes that have limited stability outside the cell, possibly owing to dissociation. Hence, they are generally employed in whole cell processes. We reasoned that CLEA formation could have a beneficial effect by holding the catalytically active multimer together, analogous to that observed with catalase (see above). This indeed proved to be the case. As noted above, a CLEA prepared from a NHase isolated from an alkaliphilic bacterium showed much improved operational stability, and excellent activity was observed in the conversion of acrylonitrile to acrylamide and a variety of aliphatic nitriles (van Pelt et al. 2008b).

\section{Hydroxynitrile lyase CLEAs}

Hydroxynitrile lyases (E.C. 4.1.2.10) catalyze the enantioselective hydrocyanation of a wide range of aldehydes. For example, CLEAs prepared from the $(R)$-specific oxynitrilase from almonds, P. amygdalus ( $\mathrm{PaHnL})$, by cross-linking with glutaraldehyde or dextran polyaldehyde, were highly effective catalysts for the hydrocyanation of aldehydes under microaqueous conditions and could be recycled several times without loss of activity (van Langen et al. 2005). Similarly, a CLEA of the relatively unknown $(R)$ selective hydroxynitrile lyase from Linus usitatissimum ( $L u \mathrm{HNL}$ ) was used in the conversion of butanone to the $R$ cyanohydrin with $87 \%$ ee (Cabirol et al. 2008). CLEAs were also prepared from the $(S)$-specific hydroxynitrile lyases from Manihot esculenta and Hevea brasiliensis (Cabirol et al. 2006; Chmura et al. 2006). High enantioselectivities were obtained in the hydrocyanation of pyridine3-aldehyde (Fig. 8) using CLEAs of the $(R)$ or (S)-specific hydroxynitrilases (Roberge et al. 2007). The latter is a difficult substrate for enantioselective hydrocyanation owing to the relatively facile non-enzymatic background reaction as a result of the electron-attracting properties of the pyridine ring. The high enantioselectivities observed with the $\mathrm{HnL}-$ CLEAs could not be obtained with the free enzyme or any other immobilized form.

\section{Catalytic cascades and combi-CLEAs}

Catalytic cascade processes have numerous potential benefits in the context of industrial production (Bruggink et al. 2003). They involve fewer unit operations, less reactor volume, higher volumetric and space-time yields, and shorter cycle times and generate less waste compared to more conventional multi-step processes. By coupling steps together, unfavorable equilibria can be driven towards completion. Biocatalytic processes are ideally suited for combination in cascade processes because they are performed under roughly the same conditions of temperature and pressure. We have already mentioned (see earlier) the co-immobilization of catalase and glucose oxidase or galactose oxidase in combi-CLEAs. We recently used (Chmura et al., manuscript in preparation; Mateo et al. 2006) a combi-CLEA containing the $M$. esculenta hydroxynitrile lyase, in combination with a nitrilase and an amidase, to catalyze the formation of $S$-mandelic acid in $99 \%$ ee at $96 \%$ benzaldehyde conversion (Fig. 9). The enantioselectivity is provided by the HNL, and in situ conversion by the nitrilase serves to drive the equilibrium of the first step towards product. The amidase was necessary to catalyze the hydrolysis of the amide byproduct formed in the nitrilase step. Similarly, a combi-CLEA comprising the $M$. esculenta hydroxynitrile lyase and the

Fig. 9 One-pot conversion of benzaldehyde to $(S)$-mandelic acid with a triple decker CLEA

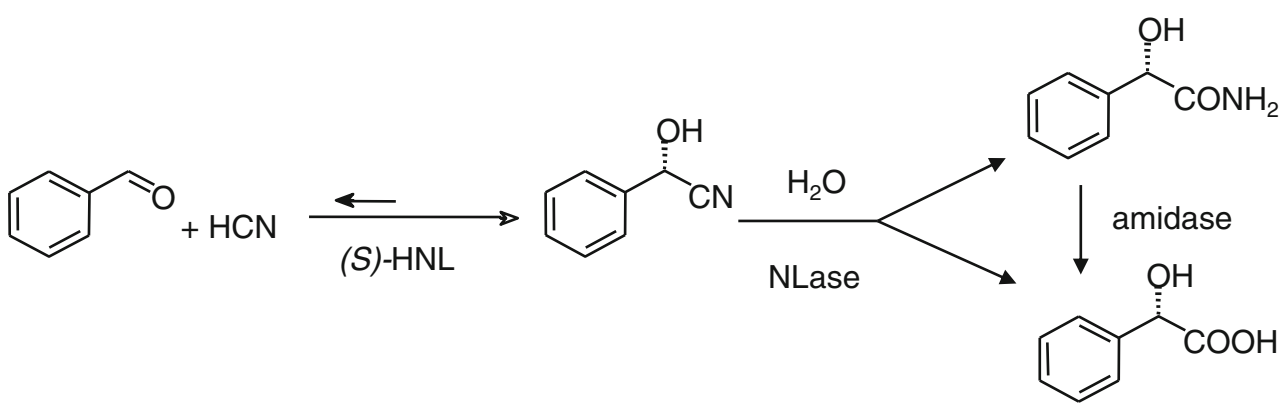

Conv. $96 \%$ / ee $>99 \%$ 
alkaliphilic nitrile hydratase from $N$. akaliphilus (EC 4.2.1.84) catalyzed the one-pot conversion of aldehydes to (S)- $\alpha$-hydroxycarboxylic acid amides (van Pelt et al. 2009).

\section{Summary and future outlook}

Enzymes have many attractive features in the context of sustainable technologies. They are derived from renewable materials, are biodegradable, and catalyze a wide variety of processes in water at ambient temperature and pressure, without the need for protection/deprotection and activation steps that characterize many traditional syntheses. However, for commercial viability, it is often necessary to immobilize the enzyme in order to achieve improved stability and facilitate recovery and reuse. The CLEA methodology has several additional advantages compared to alternative methods for immobilization of enzymes. It is simple and does not require highly pure enzymes. Indeed, it constitutes a combination of purification and immobilization into one operation and, in the optimum scenario, can be performed with cell lysates obtained directly from fermentation broth, which translates to low costs and short time to market. It has a broad scope and affords robust, recyclable catalysts that exhibit high activity retention, enhanced thermal stability, better tolerance to organic solvents, and enhanced resistance to autoproteolysis. The technique has been successfully applied to a broad range of enzymes. CombiCLEAs containing two or more enzymes have been prepared and used advantageously in catalytic cascade processes. Indeed, we have only been scratching at the surface regarding the potential applications of CLEAs. We believe that CLEAs will be widely applied in the future development of sustainable products and processes for the chemical and allied industries.

Open Access This article is distributed under the terms of the Creative Commons Attribution Noncommercial License which permits any noncommercial use, distribution, and reproduction in any medium, provided the original author(s) and source are credited.

\section{References}

Aytar BS, Bakir U (2008) Preparation of cross-linked tyrosinase aggregates. Process Biochem 43:125-131

Bankar SB, Bule MV, Singhal L, Ananthanarayan RS (2009) Glucose oxidase-an overview. Biotechnol Adv 27:489-501

Bode ML, van Rantwijk F, Sheldon RA (2003) Crude aminoacylase from Aspergillus sp. is a mixture of hydrolases. Biotechnol Bioeng 84:710-713

Bruggink A, Schoevaart R, Kieboom T (2003) Concepts of nature in organic synthesis: cascade catalysis and multistep conversions in concert. Org Proc Res Dev 7:622-640
Burgess RR (2009) Protein precipitation techniques. Methods Enzymol 463:331-342

Cabana H, Jones JP, Agathos SN (2007) Preparation and characterization of cross-linked laccase aggregates and their application to the elimination of endocrine disrupting chemicals. J Biotechnol 132:23-31

Cabirol FL, Hanefeld U, Sheldon RA (2006) Immobilized hydroxynitrile lyases for enantioselective synthesis of cyanohydrins: sol-gels and cross-linked enzyme aggregates. Adv Synth Catal 348:1645-1654

Cabirol FL, Tan PL, Tay B, Cheng S, Hanefeld U, Sheldon RA (2008) Linum usitatissimum hydroxynitrile lyase cross-linked enzyme aggregates: a recyclable enantioselective catalyst. Adv Synth Catal 350:2329-2338

Cao L, van Rantwijk F, Sheldon RA (2000) Cross-linked enzyme aggregates: a simple and effective method for the immobilization of penicillin acylase. Org Lett 2:1361-1364

Cao L, van Langen LM, van Rantwijk F, Sheldon RA (2001) Cross-linked aggregates of penicillin acylase: robust catalysts for the synthesis of beta-lactam antibiotics. J Mol Catal B: Enzym 11:665-670

Cao L, van Langen L, Sheldon RA (2003) Immobilised enzymes: carrier-bound or carrier-free? Curr Opin Biotechnol 14:387-394

Chmura A, van der Kraan GM, Kielar F, van Langen LM, van Rantwijk F, Sheldon RA (2006) Cross-linked aggregates of the hydroxynitrile lyase from Manihot esculenta: highly active and robust biocatalysts. Adv Synth Catal 348:1655-1661

Dalal S, Kapoor M, Gupta MN (2007) Preparation and characterization of combi-CLEAs catalyzing multiple non-cascade reactions. J Mol Catal B: Enzym 44:128-132

DiCosimo R (2006) In: Patel RN (ed) Biocatalysis in the pharmaceutical and biotechnology industries. CRC Press, Boca Raton, pp 1-26

Dong T, Zhao L, Huang Y, Tan X (2010) Preparation of cross-linked aggregates of aminoacylase from Aspergillus melleus by using bovine serum albumin as an inert additive. Bioresource Technol 101:6569-6571

Englard S, Seifter S (1990) Precipitation techniques. Methods Enzymol 182:287-300

Hara P, Hanefeld U, Kanerva LT (2008) Sol-gels and cross-linked aggregates of lipase PS from Burkholderia cepacia and their application in dry organic solvents. J Mol Catal B: Enzym 50:80-86

Hickey AM, Marle L, McCreedy T, Watts P, Greenway GM, Littlechild JA (2007) Immobilization of thermophilic enzymes in miniaturized flow reactors. Biochem Soc Trans 35:1621-1623

Hobbs HR, Kondor B, Stephenson P, Sheldon RA, Thomas NR, Poliakoff M (2006) Continuous kinetic resolution catalysed by cross-linked enzyme aggregates in supercritical CO2. Green Chem 8(9):816-821

Illanes A, Wilson L, Caballero E, Fernandez-Lafuente R, Guisan JM (2006) Crosslinked penicillin acylase aggregates for synthesis of beta-lactam antibiotics in organic medium. Appl Biochem Biotechnol 133(3):189-202

Illanes A, Wilson L, Altamirano C, Cabrera Z, Alvarez L, Aguirre C (2007) Production of cephalexin in organic medium at high substrate concentrations with CLEA of penicillin acylase and PGA-450. Enzym Microb Technol 40:195-203

Illanes A, Wilson L, Aguirre C (2009) Synthesis of cephalexin in aqueous medium with carrier-bound and carrier-free penicillin acylase biocatalysts. Appl Biochem Biotechnol 157:98-110

Kartal F, Janssen MHA, Hollmann F, Sheldon RA, Kilınc A (2011) Improved esterification activity of Candida rugosa lipase in organic solvent by immobilization as cross-linked enzyme aggregates (CLEAs). J Mol Catal B: Enzym 71:85-89

Kumari V, Shah S, Gupta MN (2007) Preparation of biodiesel by lipasecatalyzed transesterification of high free fatty acid containing oil from Madhuca indica. Energy \& Fuels 21:368-372

Lopez-Gallego F, Betancor L, Hidalgo A, Alonso N, Fernandez-Lafuenta R, Guisan JM (2005) Co-aggregation of enzymes and polyethyleneimine: a simple method to prepare stable and immobilized derivatives of glutaryl acylase. Biomacromol 6:1839-1842 
Lopez-Serrano P, Cao L, van Rantwijk F, Sheldon RA (2002) Cross-linked enzyme aggregates with enhanced activity: application to lipases. Biotechnol Lett 24:1379-1383

Luetz S, Giver L, Lalonde J (2008) Engineered enzymes for chemicals production. Biotechnol Bioeng 101:647-653

Majumder AB, Mondal K, Singh TP, Gupta MN (2008) Designing cross-linked lipase aggregates for optimum performance as biocatalysts. Biocatal Biotrans 26:235-238

Margolin A, Navia MA (2001) Protein crystals as novel catalytic materials. Angew Chem Int Ed 40:2204-2222

Mateo C, Palomo JM, van Langen LM, van Rantwijk F, Sheldon RA (2004) A new, mild crosslinking methodology to prepare cross-linked enzyme aggregates. Biotechnol Bioeng 8:273-276

Mateo C, Chmura A, Rustler S, van Rantwijk F, Stolz A, Sheldon RA (2006) Synthesis of enantiomerically pure (S)-mandelic acid using an oxynitrilase-nitrilase bienzymatic cascade: a nitrilase surprisingly shows nitrile hydratase activity. Tetrahedron Asymm 17:320-324

Matijosyte I, Arends IWCE, de Vries S, Sheldon RA (2010) Preparation and use of cross-linked enzyme aggregates (CLEAs) of laccases. J Mol Catal B: Enzym 62:142-148

Migneault I, Dartiquenave C, Bertrand MJ, Waldron KC (2004) Glutaraldehyde: behaviour in aqueous solution, reaction with proteins, and application to enzyme croslinking. Biotechniques 37(5):790-796-798-802

Montoro-Garcia S, Gil-Ortiz F, Navarro-Fernandez J, Rubio V, GarciaCarmona F, Sanchez-Ferrer A (2009) Improved cross-linked enzyme aggregates for the production of desacetyl beta-lactam antibiotics intermediates. Bioresource Technol 101:331-336

Nuijens T, Cusan C, van Dooren TJGM, Moody HM, Merkx R, Kruijtzer JAW, Rijkers DTS, Liskamp RMJ, Quaedflieg PJLM (2010) Fully enzymatic peptide synthesis using C-terminal tert-butyl ester interconversion. Adv Synth Catal 352:2399-2404

Özdemirhan D, Sezer S, Sönmez Y (2008) Enzyme catalyzed resolution of aromatic ring fused cyclic tertiary alcohols. Tetrahedron Asymm 19:2717-2722

Park HJ, Uhm KN, Kim HK (2010) Biotransformation of amides to acids using a co-cross-linked enzyme aggregate of Rhodococcus erythropolis amidase. J Microbiol Biotechnol 20:325-331

Pchelintsev NA, Youshko MI, Svedas VK (2009) Quantitative characteristic of the catalytic properties and microstructure of cross-linked enzyme aggregates of penicillin acylase. J Mol Catal B: Enzym 56:202-207

Perez DI, van Rantwijk F, Sheldon RA (2009) Cross-linked enzyme aggregates of chloroperoxidase: synthesis, optimization and characterization. Adv Synth Catal 351:2133-2139

Roberge C, Fleitz F, Pollard D, Devine P (2007) Asymmetric synthesis of cyanohydrin derived from pyridine aldehyde with cross-linked aggregates of hydroxynitrile lyases. Tetrahedron Lett 48:1473-1477

Schoevaart R, Wolbers MW, Golubovic M, Ottens M, Kieboom APG, van Rantwijk F, van der Wielen LAM, Sheldon RA (2004) Preparation, optimization, and structures of cross-linked enzyme aggregates (CLEAs). Biotechnol Bioeng 87:754-762

Schoevaart WRK, van Langen LM, van den Dool RTM, Boumans JWL (2006) WO 2006/046865 A2, to CLEA Technologies

Shah S, Sharma A, Gupta MN (2006) Preparation of cross-linked enzyme aggregates by using bovine serum albumin as a proteic feeder. Anal Biochem 351:207-213

Sheldon RA (2007a) Enzyme immobilization: the quest for optimum performance. Adv Synth Catal 349:1289-1307
Sheldon RA (2007b) Cross-linked enzyme aggregates (CLEAs): stable and recyclable biocatalysts. Biochem Soc Trans 35:1583-1587

Sheldon RA (2011) Cross-linked enzyme aggregates as industrial biocatalysts. Org Proc Res Dev 15:213-223

Skovgaard J, Bak CA, Snabe T, Sutherland DS, Laursen BS, Kragh KM, Besenbacher F, Poulsen CH, Shipovskov S (2010) Implementation of cross-linked enzyme aggregates of proteases for marine paint applications. J Mater Chem 2:7626-7629

Sorgedrager MJ, Verdoes D, van der Meer H, Sheldon RA (2008) Cross-linked enzyme aggregates in a membrane slurry reactor. Continuous production of 6-APA by enzymatic hydrolysis of penicillin. Chimica Oggi/Chemistry Today (Focus on Biocatalysis) 26:23-25

Sulek F, Perez Fernandez D, Kneza Z, Habulina M, Sheldon RA (2011) Immobilization of horseradish peroxidase as crosslinked enzyme aggregates (CLEAs). Process Biochem 46:765-769

Turner NJ (2009) Directed evolution drives the next generation of biocatalysts. Nature Chem Biol 5:567-577

Vafiadi C, Topakas E, Christakopoulos P (2008) Preparation of multipurpose cross-linked enzyme aggregates and their application to production of alkyl ferulates. J Mol Catal B: Enzym $54: 35-41$

van Langen LM, Selassa RP, van Rantwijk F, Sheldon RA (2005) Cross-linked aggregates of (R)-oxynitrilase: a stable, recyclable biocatalyst for enantioselective hydrocyanation. Org Lett 7:327329

van Pelt S, van Rantwijk F, Sheldon RA (2008a) Nitrilehydratases in synthesis. Chimica Oggi/Chem Today 26(3):2-4

van Pelt S, Quignard S, Kubac D, Sorokin DY, van Rantwijk F, Sheldon RA (2008b) Nitrile hydratase CLEAs: the immobilization and stabilization of an industrially important enzyme. Green Chem 10:395-400

van Pelt S, van Rantwijk F, Sheldon RA (2009) Synthesis of aliphatic $(S)-\alpha$-hydroxycarboxylic amides using a one-pot bienzymatic cascade of immobilised oxynitrilase and nitrile hydratase. Adv Synth Catal 351:397-404

Walt DR, Agayn VI (1994) The chemistry of enzyme and protein immobilization with glutaraldehyde. Trends Anal Chem 13 (10):425-430

Wilson L, Illanes A, Pessela BCC, Abian O, Fernandez-Lafuenta R, Guisan JM (2004a) Encapsulation of crosslinked penicillin $g$ acylase aggregates in lentikats: evaluation of a novel biocatalyst in organic media. Biotechnol Bioeng 86:555-562

Wilson L, Betancor L, Fernandez-Lorente G, Fuentes M, Hidalgo A, Guisan JM, Pessela BCC, Fernandez-Lafuente R (2004b) Crosslinked aggregates of multimeric enzymes: a simple and efficient methodology to stabilize their quaternary structure. Biomacromol 5:814-817

Wilson L, Illanes A, Soler L, Henriquez MJ (2009) Effect of the degree of cross-linking on the properties of different CLEAs of penicillin acylase. Process Biochem 44:322-326

Yu HW, Chen H, Wang X, Yang YY, Ching CB (2006) Crosslinked enzyme aggregates (CLEAs) with controlled particles: application to Candida rugosa lipase. J Mol Catal B: Enzym 43:124-127

Zhao L, Zheng L, Gao G, Jia F, Cao S (2008) Resolution of N-(2ethyl-6-methylphenyl) alanine via cross-linked aggregates of Pseudomonas sp. lipase. J Mol Catal B: Enzym 54:7-12 